\title{
The Role of Machine Learning and Data Mining Techniques in Predicting Students' Academic Performance
}

\author{
Dr. Aliyu Y. Rufai \\ Department of Computer \\ Science, Kebbi State \\ University of Science and \\ technology. Aleiro, kebbi \\ State, Nigera
}

\author{
Dr.Hassan U. Suru \\ Department of Computer \\ Science, Kebbi State \\ University of Science and \\ technology. Aleiro, kebbi \\ State, Nigeria
}

\author{
James Afrifa \\ Department of Computer \\ Science, Adamawa State \\ Polytechnic, Yola, Nigeria
}

\begin{abstract}
The advancement in Information Technology makes it easier and cheaper to collect large amounts of data, but if this data is not further analyzed, it remains only huge amounts of data. These large amounts of data set have motivated research and development in various fields to extract meaningful information with a view of analyzing it to solve complex problem. With new methods and techniques, data can be analyze and be of great advantage. Data mining and machine learning are two computing disciplines that enable analysis of large data sets using different techniques. This paper gave an overview of several applications using these disciplines in education, with focus on student's academic performance prediction. Early prediction of students' performance is useful in taking early action of improving learning outcome. The perfect methods for this are machine learning and data mining. This paper also discusses special use of data mining in education, called educational data mining. Educational Data Mining (EDM) uses different methods and techniques from machine learning, statistics, data mining and data analysis, to analyze data collected during teaching and learning. The goal of this paper is to introduce the role of machine learning and data mining in predicting student's academic performance and to present its applications and benefits
\end{abstract}

Keywords: Data, Educational Data Mining, Machine Learning, Data Mining, Education, Students' academic performance,

\section{INTRODUCTION}

Education plays a very vital role in human resource development and also in the society at large (Pinheiro, Wangenge-ouma, Balbaceusky \& Cai, 2015). Student's academic performance determines the quality of education in a higher institution of learning. Student's performance plays an essential role in producing good quality graduates that in future will be responsible for the country's development. The performance of the student is one of the most important aspects of every educational institution. Predicting student's academic performance is one of the most important steps towards efficient education while understanding student performance is essential for the establishment of a student centric learning environment. The definition of student's academic success is based on individual perceptions and sometimes most often misused within educational research. However, the study of York, Gibson \& Rankin (2015) suggested that the definition of academic success is made up of six components:

(1) Academic achievement, which is nearly entirely measured with course grades and grade point average (GPA), (2) Satisfaction, which is often captured either by course evaluation or institutional surveys, (3) Persistence, which is measured by retention between particular years of college and degree attainment rates, (4) Acquisition of skills and competencies, which can be measured by assignments and course evaluations, (5) Attainment of 
learning objectives, which can also be measured by assignments and course evaluations, and finally (6) Career success, which can be determined by job attainment rates, promotion histories, career satisfaction and professional goal attainment.

Another crucial requirement for maximizing students' success is the identification of the factors that affects students' academic performance. Identification of these factors could assist in achieving the highest level of quality education (Yassein, Gaffer, Helali \& Mohomed, 2017). These factors could be useful in decision making concerning student's academic performance.

Poortman \& Schildkamp (2016) Suggested that Data - base decision can contribute to increased student learning and achievements.

With advancement in Information Technology (IT) development and lower prices, tertiary institutions start to collect a huge amount of data about their students. These data can be further analyzed with data mining methods and techniques.

Data mining tools, methods and techniques, allow you to analyze data and find hidden patterns and information. Data mining is said to be the most robust methodology mostly used for assessing useful information from the data warehouse ( Salloum, El-emran, Addallah \& Shaalan, 2017). Data miming can improve decision making by predicting hidden information through extraction method ( Salloum, Mhamdi, Aliemran \& Shaalan, 2017) . Data mining is used to detect patterns and relationships in data to improve decision-making processes. Data mining is an interdisciplinary field that brings together techniques from statistics, artificial intelligence, neural networks, database systems, machine learning, pattern recognition, data visualization, knowledge acquisition and information theory (Arunachalam \& Velmurugan, 2018) (Sumathi \& Sivanandam, 2013). The practice of Data Mining methods applied to educational data is known as Educational Data Mining (EDM) (Baker \& Yacef 2009). It is an interdisciplinary area that brings together techniques from statistics, artificial intelligence, database systems, machine learning, pattern recognition, data visualization, knowledge acquisition and information theory (Sumathi and Sivanandam, 2006) to find useful patterns and, thus, help understand students' behavior and how they learn It is drawn from a variety of domains, including Data Mining, Machine Learning, psychometrics and other areas of statistics, information visualization, and computational modeling (Romero and Ventura 2007). The education system has become more balanced due to the improved mining application ( Romero, Ventura \& Garcia, 2008)

\section{EDUCATIONAL DATA MINING (EDM)}

Educational institutions collects huge amount of data and stores them. The amount of data collected and stored are growing too big and educational data analysis could not be performed manually anymore. One of the elements of data mining is educational data mining (EDM), the key focus of which is on developing models for extracting hidden knowledge from the student's data, which if use will enhance student's academic performance. Educational data mining may also be considered as a new model that is part of the prevailing education system, which is able to generate positive interaction with different parts of the system. This will enable it to eventually attain the objective of enhancing teaching ( Iie-Haiyan, Biac \& Yuan, 2017). The goal of EDM is to improve the educational process and to explain educational strategies for better decision making (Silva \& Fonseca, 2017)

Educational Data Mining (EDM) is defined as the application of techniques of traditional data mining to educational data analysis, with the objective of obtaining solutions to problems in the field of education ( Baker \& Yacef, 2009). There are certain EDM applications that include the formulation of e-learning systems ( Lara, Lizcano, Martinez, Pazos \& Riera, 2014), clustering educational data (Chakrabary, Chakma \& Mukherjee, 2016) as well as making student performance predictions( Chauhan, Shah, Karn, \& Dalal,2019). Several kinds of techniques are presently quite popular in educational data mining, which are part of the following categories: sequential pattern, clustering, prediction, classification, machine learning models and association rule analysis. 


\subsection{Educational Data Mining Process}

There are series of phases involved in the EDM, however, the EDM process has four main basic phases. The first among them is the Problem definition Phase in which a specific problem is translated into a data mining problem. This phase formulated the project goal and objectives, as well as the main research questions. The second phase of the EDM process is the data preparation and gathering phase, and it is most timeconsuming phase. It can take up to $80 \%$ of all analysis time. Data quality is a major challenge in data mining (Blake and Mangiameli, 2011). In this phase, source data must be identified, cleaned and formatted in prespecified format. Once that is done, the next phase is the Modeling and Evaluating phase in which the parameters are set to optimal values and different modeling techniques are selected and applied. The last phase is the deployment phase in which the results of data mining are organized and presented through graphs and reports.

\section{MACHINE LEARNING AND DATA MINING}

This study focused on the role of Machine learning and Data mining in predicting student's performance. ML and DM are mostly confusing because of similar meanings, so they have a meaningful similarity. Founder of machine learning, Arthur Samuel, describes it as an area of research which gives the ability to learn without being explicitly programmed. ML interacts with learning the pattern recognition and computational learning theory in artificial intelligence. ML is older than DM. In the recent days, the term data mining is extra popular than its sibling machine learning which can be the reason for some scholars to actually highlight their study for data mining than machine learning, so in this study, machine learning and data mining are discussed together. Every DM and ML process involves six main steps. In the First, there is a Business Understanding in which a specific problem is translated into a data mining problem. The second step is Data Understanding, which starts with data collected from all applicable data sources. In this step, data load and data integration are done. Data visualization tools are often used in this step to explore the properties of the data. The most important step is Data Preparation, and it can take enormous amounts of time depending on the amount of data analyzed and the number of data sources (Blake and Mangiameli, 2011). Data quality is a major challenge in data mining. The final data set must be cleaned, formatted and constructed into a specific form. In the Modelling and Evaluation step, mathematical models are used to find patterns in the data using sophisticated data tools and parameters are calibrated to optimal values. The last step is Deployment in which the results of data mining are presented (Oracle, 2019).

Data mining and machine learning is basically classified into supervised, un-supervised and reinforcement approaches.

Supervised Learning: whereby the machine is provided with labeled data for both input as well as expected output during its training and the supervised learning algorithm generates a mapping function that can identify the expected output for a given input. The training process continues till the algorithm reaches the preferred level of accuracy. One of the standardized goals of supervised learning is to make the computer learn a classification system; therefore, it is commonly used to solve classification problems. For example, the machine could be trained to classify a spam email from a valid e-mail, already being used by Google for Gmail spam filtering. Nearest neighbor, Naïve Bayes, Decision Trees, Linear Regression, Support Vector Machines and Neural Networks are a few of the most common algorithms that are included under this category.

Unsupervised Learning: whereby the machine is provided with unlabeled and unclassified input dataset and the unsupervised learning algorithm generates a function to identify hidden structures in the given dataset as per the patterns, similarities and differences that exist among data without any former training. There is no assessment of the level of accuracy of the structure identified by the machine. One of the major focuses of unsupervised learning algorithms could be clustering and association problems. A 
few of the commonly used unsupervised learning algorithms are k-means algorithm for clustering and Apriori algorithm for association problems.

Reinforcement Learning: the machine is exposed to an environment where it takes decisions on a trial and error basis and learns from its own actions and past experiences. For every correct decision the machine receives a reward feedback from the environment that acts like a reinforcement signal and the information about the rewarded state-action pair is stored. Later on the machine iterates the rewarded behavior whenever faced with a similar situation. Reinforcement learning algorithms have their usage in domains where strategic decision making is the key to success like Self Driving Cars. Few of the most commonly used reinforcement learning algorithms are QLearning and Markov Decision processes (Puget, 2016).

\section{DATA MINING AND MACHINE LEARNING TECHNIQUES FOR PREDICTING STUDENT'S PERFORMANCE.}

The ability for an institution to predict results might help students develop a good understanding of how well or bad they would perform in a course and then can take steps accordingly.

Early prediction of students' performance is useful in taking early action of improving learning outcome. Predicting a student's performance from past academic data is one of the most popular applications of educational data mining and, therefore, it is a valuable source of information that can be used to improve students' performance (Buenaño-Fernández, Gil \& Luján-Mora, 2019).

This section discussed the various techniques in DM and ML that are used in predicting students' performance. There are many various DM and ML d methods to build a predictive model for student's performance. The most commonly used method is classification. Among classification algorithms are Neural Networks, Decision Trees, Naïve Bayes, Support Vector Machine and KNearest Neighbor (Shahiri and Husain, 2015).

\subsection{Classification Algorithm}

Classification is a data mining technique that segments data in a collection to target categories or classes. It helps in analyzing data and predicting outcomes. The goal of classification is to accurately predict the target class or each case in the data. The classifier training algorithm uses pre-classified examples for determining the set of parameters required for classification (Oracle, 2019). In the educational sector, this technique is often used for classifying students based on some characteristics such as age, gender, grades, knowledge, academic achievements, motivation, behavior, demographic or geographic characteristics, etc

\subsection{Decision tree}

A decision tree is a decision support tool that uses a treeshaped graph or model for classification. It is a supervised learning method. Each internal node represents a test on an attribute, each branch represents an outcome of the test, and each leaf node represents a class which is a decision after computing all attributes. The paths from the root to leaf are classification rules (Vidal et al, 2014). Their greatest advantage is stability and easy interpretation. Because of their simplicity, they are suitable for solving a different kind of problems in a broad range of industries such as financial, business, healthcare, education, energy, engineering, pharmaceutical, law, etc

\subsection{Artificial Neural Networks.}

Artificial neural network inspired by the human brain includes huge number of neurons with every neuron having an input and output with an activation function. Commonly, neural network is based on the layer approach ; the first layer is the input layer, the last layer is the output layer, and the other layers are known as hidden layer

\subsection{Naive Bayes.}

It is a simple probabilistic classifier [33]based on the Bayes theorem. It gives a way to calculate the future probability $\mathrm{P}(\mathrm{c}-\mathrm{x})$, from $\mathrm{P}(\mathrm{c}), \mathrm{P}(\mathrm{x})$, and $\mathrm{P}(\mathrm{x}-\mathrm{c})$. It assumes that the result of the value of the predictor (x) on the given class (c) is liberated than the other predictors. The theory is known as "class conditional independence."

\subsection{Support Vector Machine.}


SVM is as supervised ML algorithm to help for classification/regression problems. It based on searching an unraveling hyper plane in the feature space in two classes in such a way that the space between the hyper plane and the nearest data point of every class is maximized. This method depends upon reduced classification threat somewhat that is an ideal classification. SVM has good reputation for its generalization ability and binary classifier, and multiclass classification is understood by making an SVM for every pair of class.

\subsection{Regression Analysis}

Prediction refers to calculated assumptions for certain events made based on available processed data. Regression technique can be used for prediction, to model the relationship between one or more independent variables and dependent variables. Independent variables are attributes already known and response variables are what we want to predict (Bhatnagar, 2013). It has many applications in business planning, trend analysis, financial forecasting, time series prediction, trend analysis,etc. In the educational sector, it is used for the prediction of students' academic performance, prediction of enrolled students, prediction of the final grade, prediction of drop-outs, etc.

\section{TOOLS FOR DM AND ML}

There are four free and most famous software including RStudio, RapidMiner, Pandas, StatsModels, and Scikitlearn (python libraries) and Weka. RStudio is an IDE for analytical computing and graphics. It is a core programming language known as Rlanguage. It has console, code highlighting editor for the direct lunching code with utility for history, debugging, and so on. RStudio has different edition including free of cost, business use, and web application platform. After Rfunctionality, many high-level scripting languages like python are used by students R especially for sample regression and correlation analysis. RapidMiner is a good tool which provides the platform for data mining, machine learning, text mining, predictive analysis, and soon. It can create statistical workflow getting data from many data sources. RapidMiner is used for business, commercial, research, education, and training purposes. It is compatible with the stages of data mining like data preprocessing, creating model, graphical result, and so on. It is an excellent educational tool in the field of data science. Nowadays, Python is the most popular multipurpose programming language. It has packages, libraries, and framework for data analysis and visualization, which are related tasks. Pandas, Stats Models, and Scikitlearn are the three open-source libraries. It can be best to use all three libraries in IPython. "Pandas" is an opensource library that gives high performance easy to use data analysis tools for the Python language; "Statsmodel" is also the Python library which facilitates programmer to data explore, estimate analytical model, and perform statistical test."Scikit-learn" is another open-source machine learning library for Python. It has many classifications, regression, random forest, K-means, and so on. It is a highly useful library to explore data and estimate analytical model and test. Weka is used to implement algorithms related to data mining and machine learning based on Java. It has tools for data preprocessing, regression analysis, clustering, classification methods, association rules, and so on. Weka is free to use and has user-friendly GUI (Bilal, Wang, \& Zain, 2018).

\section{RELATED WORK}

The need to predicting student academic performance has become a crucial factor in improving the quality of education; assist the students' academic performance as well as providing the teachers more options when training their students. Educational data mining is a young research area which is becoming increasingly popular due to its potential. Educational data can be used to assist instructors, to improve curriculums, to understand students' behavior, to improve teaching process, to improve e-learning systems, to identify reasons for dropping out, to support decision making and improve students learning behavior (Romero \& Ventura, 2010). In recent times, there are many works been published related to this subject matter.

Anderson, Boodhwani \& Baker (2019) conducted a study to predict graduation at a public R1 university using linear 
support vector machines, decision trees, logistic regression, and stochastic gradient descent binary classifiers. The study used a data set of over 14,000 students from six Fall cohorts, containing 104 features, drawn from pre-existing university data. The prediction accuracy was as follows; decision tree 0.786, linear SVM 0.801, logistic regression, 0.810 and stochastic gradient descent binary classifiers 0.824 .

According Sedkaoui \& Khelfaoui (2019), an analysis of the relationship between big data and educational environments has been presented. The work focuses on the different methods, techniques, tools, and big data algorithms that can be used in the educational context in order to understand the benefits and impact that can cause in the teaching and learning process. The discussion generated in their study suggests that the incorporation of an approach based on big data is of crucial importance. This approach can contribute significantly in the improvement of the learning process, for its implementation must be correctly aligned with the learning needs and the educational strategies.

Devasia et al. (2016) examine various data mining techniques for the prediction of students' performance. They used all student admission details, course details, subject details, student marks details, attendance details and student's academic history as input. The results of their paper show that Naive Bayesian algorithm is more accurate than other methods like Regression, Decision Tree, Neural networks etc., for comparison and prediction.

Oancea, Dragoescu, \& Ciucu (2013) used the classification power of a neural network to predict the students' results measured by the grade point average in the first year of study. The input variables were:type of the study program (part-time or full-time education),gender, high-school graduation average, age and difference in years from the moment the student graduates high-school until he/she enrolls at university. The goal of their research was to help university management in order to take early action to avoid the phenomenon of leaving education.

\section{The Role/Application of DM and ML}

The role and applications of DM and ML are numerous. There are many research papers and studies regarding the use and applications of $\mathrm{ML}$ and $\mathrm{DL}$ in predicting student's academic performance education. The most common use of the two terms include: improving the process of studying, improving course completion, supporting students in course selection, students' profiling, finding problems leading to dropping out, students' targeting, curriculum development, predicting student's performance and as a support for decision-making at student enrolment.

Predicting a student's performance from past academic data is one of the most popular applications of educational data mining and, therefore, it is a valuable source of information that can be used to improve students' performance (Buenaño-Fernández, Gil \& Luján-Mora, 2019). This is where Data Mining and Machine Learning come in. Machine Learning is a set of techniques that gives computers the ability to learn without the intervention of human programming ( Navamani \& Kannammal, 2015).

The application of machine learning techniques to predicting students' performance, based on their background information and their in-term performance has proved to be a helpful tool for foreseeing poor and good performances in various levels of education (Soni, Kumar, Kaur \& Hemavath, 2018). Machine learning offers an advantage over traditional forms of statistical analysis, placing emphasis on predictive performance over provable theoretical properties and priori super-population assumptions. Thereby tutors are enabled to timely help the weakest ones, but also, to promote the strongest thus improving learning.

Yukselturk, Ozekes \& Türel (2014) experimented with four algorithms: k-nearest neighbors, decision trees, the naive Bayes classifier and artificial neural networks, to classify students who dropped out of school. The data set was collected by administering an online test for 189 students enrolled in 2007 to 2009 . The machine learning algorithms were trained and tested using the 10-fold cross-validation technique. The best results were obtained using 3-nearest neighbors and decision trees, with an accuracy of $87 \%$ and $79.7 \%$ respectively. These results were useful since they permitted prediction of student dropout in the online program data set. Finally, the authors concluded that data mining methods might 
help to predict different reasons why students decide to drop out before finishing their study programs.

The application of machine learning techniques to tackled problem was introduced by Kakavand, Mokfi \& Tarokh (2014), with the purpose of predicting student loyalty using decision trees. The authors researched the external factors that may generate loyalty, in order to identify students who have decided to continue studying, and thus the university may invest in them and increase its educational quality. The experiments were performed using a data set of 135 instances for training, 33 for testing and 35 for validation, with 14 attributes per instance (gender, age, and income, among others). The best result was obtained using the CARTdecision tree algorithm, with $94 \%$ accuracy.

In another research, Data Mining: A prediction for performance improvement using classification Bhardwaj and Pal (2012) used Bayes classification for the construction of a prediction model to identify the difference between high learners and slow learners.

Kovačić (2012) examined the socio-demographic variables (age, gender, ethnicity, education, work status, and disability) and study environment (course program and course block) that may help in identifying successful and unsuccessful students. This research concluded that classifying students based on pre-enrolment information can help to identify students at-risk of dropping the course and suggest advising and mentoring programs to make them successful.

Maqsood (2013) stated that data mining can be used to report and analyze the data that can help in preparing marketing strategies for targeted students.

Kumar and Chadha (2011) presented an empirical study of the applications of data mining techniques in higher education in which they tried to identify the potential areas in which data mining techniques could be applied. They concluded that potential applications are: organization of syllabus, predicting the registration of students in an educational program, predicting student performance, detecting cheating in the online examination and identifying abnormal or erroneous values and used data mining techniques are: association analysis, classification, prediction, clustering and outlier analysis.

Kumar \& Pal (2011) used decision trees to extract a set of academic characteristics to assess students' performance. The data set consisted of 50 examples from students of the computer applications department at the VBS Purvanchal University, India. The characteristics considered included grades in previous semesters, seminar performance, general proficiency performance, and attendance. The knowledge extracted and represented by the decision tree enabled the authors to obtain if-then rules to classify the students. With this work, the authors predicted students' end-of-semester performance and identified students who needed special attention to reduce the failure rate.

Data mining was used by Ranjan \& Khalil (2008) with two main objectives: 1) planning a course for education management through new data mining applications and to explore the effects on probable changes in the recruitment and admission processes and guiding courses; and 2) ensuring quality evaluations, student performance, courses and tasks. Also, they were interested in finding patterns in how students interact with others, how the admission process is carried out, and the mechanisms in counseling to choose courses. The framework was tested to find what types of courses are interesting to certain types of students. In addition, the proposed framework had three major processes that usually occur in all management institutions, namely admissions (planning, evaluation and registration), counseling, and allocation of specialization subjects. The results were presented as a conceptual framework to adopt data mining in management in institutions. The authors concluded that data mining is useful for predicting the success of educational programs, and also understanding learning styles in order to promote proactivity in students. Oladokun, Adebanjo and Charles-Owaba (2008) proposed a neural network model to predict the performance of possible 
candidates for admission to university. Their model was based on a multilayer perceptron topology, with family history, age, and score of the entrance exam taken as input variables, among others. They reported about $74 \%$ accuracy in their results, but recommended conducting an oral interview to obtain more information to supply to their model.

\section{CONCLUSION}

This study demonstrates the role of machine learning and data mining techniques for predicting student's academic performance. The study highlighted the papers that define the use of multiple Machine Learning and Data Mining techniques for predicting student's academic performance.

Data Mining and Machine Learning within the academic context is applicable, allowing educational institutions to better allocate human and material resources, manage student performance and improve the effectiveness of performance throughout students 'education. Machine learning and Data mining techniques were developed to automatically discover hidden knowledge and recognize patterns from data. Educational data mining can be used for classifying and predicting students' performance, dropouts as well as teachers' performance.

. In this paper, we presented the benefits and applications of machine learning and data mining techniques in many educational areas. The main goal of the paper is to reveal the high potential of educational data mining applications, techniques, tools and its role in predicting student's academic performance.

\section{ACKNOWLEDMENTS}

Our thanks goes to everyone who in one way or the other has contributed to the success of this paper.

\section{REFERENCES}

1) Anderson, H., Boodhwani, A., \& Baker, R. (2019). Predicting graduation at a public R1 university. In Proceedings of the 9th International Learning Analytics and Knowledge Conference.

2) Arunachalam, A.S., \& Velmurugan, T. (2018). Analyzing student performance using evolutionaryartificial neural network algorithm. Int. J. Eng. Technol.7(2.26), 67-73

3) Baker, R. S., \& Yacef, K. (2009). The state of educational data mining in 2009: A review and future visions. JEDM| Journal of Educational Data Mining, 1(1), 3-17

4) Bhardwaj, B. K., Pal, S. (2012). Data Mining: A prediction for performance improvement using classification. Retrived 25.5.2019. from https://arxiv.org/pdf/1201.3418.pdf

5) Bhatnagar, V. (Ed.). (2013). Data mining in dynamic social networks and fuzzy systems. IGI global

6) Bilal, A., Wang, J., \& Zain, A. (2018). Role of Machine Learning and Data Mining in Internet Security: Standing State with Future Directions Journal of Computer Networks andCommunications Volume 2018, Article ID 6383145 , pageshttps://doi.org/10.1155/2018/6383145

7) Blake, R., \& Mangiameli, P. (2011), "The effects and interactions of data quality and problem complexity on classification", Data Inform, pp. 160-175

8) Buenaño-Fernández, D., Gil, D., \& Luján-Mora, S. (2019). Application of Machine Learning in Predicting Performance for Computer Engineering Students: A Case Study. Sustainability, 11(10), 2833-2851.

9) Chakraborty, B., Chakma, K., \& Mukherjee, A.(2016). A density-based clustering algorithm andexperiments on student dataset with noises using Rough set theory. In: 2016 IEEEInternational Conference on Engineering and Technology (ICETECH), pp. 431-436 
10) Chauhan, N., Shah, K., Karn, D., Dalal, J. (2019). Prediction of student's performance usingmachine learning . SSRN 3370802

11) Devasia, T., Vinushree, T. P., Hegde, V. (2016). „Prediction of students performance using Educational Data Mining“. In 2016 International Conference on Data Mining and Advanced Computing (SAPIENCE). pp. 91-95.

12) Jie, W., Hai-yan, L., Biao, C., \& Yuan, Z. (2017). Application of educational data mining on analysisof students'online learning behavior. In: 2017 2nd International Conference on Image, Vision and Computing (ICIVC), pp. 10111015

13) Kakavand, S., Mokfi, T., \& Jafar, M. (2014). Prediction the loyal student using decision tree algorithms. International Journal of Information and Communication Technology Research, 4(1), 32-37

14) Kovacic, Z. (2012). Predicting student success by mining enrolment data. Research in Higher Education, 15

15) Kumar, D.N., Raju, K.S. and Sathish, T. (2004). River Flow Forecasting Using Recurrent

a. Neural Networks. Water Resources Management, Kluner Academic, 18:143- 161

16) Kumar, B., \& Pal, S. (2011). Mining educational data to analyze students' performance. International Journalof Advanced Computer Science and Applications, 2(6), 63-60.

17) Lara, J.A., Lizcano, D., Martínez, M.A., Pazos, J., \& Riera, T. (2014). A system for knowledgediscovery in e-learning environments within the European Higher Education AreaApplication to student data from Open University of Madrid, UDIMA. Comput. Educ.72,23-36

18) Maqsood, A. M. (2013). Customer Relationship Management in B-Schools: An Overview. International Journal of Computer Sciences and Management Research, 2(4), 2108-2119.
19) Navamani, J.; Kannammal, A. (2015) Predicting performance of schools by applying data mining techniques on public examination results. Res. J. Applied Science of Engineering and Technology. 2015, 9, 262-271.

20) Oancea, B., Dragoescu, R., Ciucu, S. (2013). „Predicting students' results in higher education using a neural network", available at: at https://mpra.ub.uni-muenchen.de/72041/

21) Oladokun, V. O., Adebanjo, A. T., \& Charles-Owaba, O. E. (2008). Predicting students' academic performance using artificial neural network: a case study of an engineering course. The Pacific Journal of Science and Technology, 9(1), 72-79

22) Oracle (2019), "Data Mining Concepts", available at: http://docs.oracle.com/cd/B28359_01/datamine.1 11/b28129/process.htm\#DMCON002

23) Pinheiro, R., Wangenge-Ouma, G., Balbachevsky, E., \& Cai, Y. (2015). The role of higher education in society and the changing institutionalized features in higher education. In The Palgrave international handbook of higher education policy and governance (pp. 225-242). London: Palgrave Macmillan UK.

24) Poortman, C. L., and K. Schildkamp. (2016).“Solving Student Achievement Focused Problems witha Data Use Intervention for Teachers.’Teaching and Teacher Education60: 425-433.

25) Puget, J. F. (2016). What is Machine Learning? (IT Best Kept Secret is Optimization).

Retrieved March 12, 2021, from IBM Developer Works:

https://www.ibm.com/developerworks/communit y/blogs/jfp/entry/What Is Machine Learning?la $\underline{n g=e n}$.

26) Ranjan, J., \& Khalil, S. (2008). Conceptual framework of data mining process in management education in India: an institutional 
perspective. Information Technology Journal, 7(1), 16-23

27) Romero, C., \& Ventura, S. (2010). Educational data mining: a review of the state of the art. IEEE Transactions on Systems, Man, and Cybernetics, Part C (Applications and Reviews), 40(6), 601618

28) Romero, C., Ventura, S., \& García, E.( 2008). Data mining in course management systems: moodle case study and tutorial. Comput. Educ.51(1), 368-384

29) Salloum, S.A., Al-Emran, M., \& Shaalan, K (2017). Mining social media text: extracting knowledge from Facebook. Int. J. Comput. Digit. Syst.6(2), 73-81

30) Salloum, S.A., Mhamdi, C., Al-Emran, M., \& Shaalan, K. (2017). Analysis and classification of Arabic newspapers'Facebook pages using text mining techniques. Int. J. Inf. Technol. Lang.Stud.1(2), 8-17

31) Sedkaoui, S.; Khelfaoui, M. (2019) Understand, develop and enhance the learning process with big data. Inf. Discov. Deliv. 2019, 47, 2-16.

32) Shahiri, A. M., Husain, W. (2015). „A review on predicting student's performance using data mining techniques." Procedia Computer Science. No. 72, pp. 414-422

33) Silva, C., Fonseca, J. (2017). Educational Data Mining: a literature review. In Europe and MENA Cooperation Advances in Information and Communication Technologies (pp. 87-94).

34) Soni, A., Kumar, V., Kaur, R., \& Hemavath, D. (2018). Predicting student performance using data mining techniques. International Journal of Pure and applied Mathematics, 119(12), 221227.

35) Yassein, N. A., Gaffer, R., Helali, M., \& Mohomad, S. B. (2017). Citation: Predicting student academic performance in KSA using data mining techniques. Journal of Information Technology and Software Engineering,7(5), 213.
36) York, T. T., Gibson, C., \& Rankin, S. (2015). Defining and measuring academic success: Practical Assess-ment. Research \& Evaluation,20(5), 462

37) Yukselturk, E., Ozekes, S., \& Türel, Y. K. (2014). Predicting dropout student: an application of data mining methods in an online education program. European Journal of Open, Distance and eLearning, 17(1), 118-133 